\title{
Symbol Digit Modalities Test: Normative data for the Latin American Spanish speaking adult population
}

J.C. Arango-Lasprilla ${ }^{\mathrm{a}, \mathrm{b}, *}$, D. Rivera ${ }^{\mathrm{b}}$, G. Rodríguez ${ }^{\mathrm{c}}$, M.T. Garza $^{\mathrm{d}}$, J. Galarza-del-Angel $^{\mathrm{e}}$, W. Rodríguez ${ }^{\mathrm{f}}$, J. Velázquez-Cardoso ${ }^{\mathrm{g}}$, A. Aguayo ${ }^{\mathrm{h}}$, S. Schebela $^{\mathrm{i}}$, C. Weili ${ }^{\mathrm{j}}$, M. Longoni ${ }^{\mathrm{k}}$,

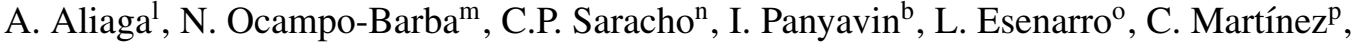
C. García de la Cadena ${ }^{\mathrm{q}}$ and P.B. Perrin ${ }^{\mathrm{r}}$ a IKERBASQUE, Basque Foundation for Science, Bilbao, Spain

${ }^{\mathrm{b}}$ Faculty of Psychology and Education, University of Deusto, Bilbao, Spain

'Hospital Clínico Quirúrgico Docente "Hermanos Ameijeiras", Havana, Cuba

${ }^{\mathrm{d}}$ Facultad de Psicología, Universidad Autónoma de Nuevo Leon, Monterrey, Mexico

${ }^{\mathrm{e}}$ Universidad Autónoma de Baja California, Mexicali, Mexico

${ }^{\mathrm{f}}$ Ponce Health Sciences University, Ponce, Puerto Rico

${ }^{\mathrm{g}}$ Instituto Nacional de Neurología y Neurocirugía MVS, Mexico City, Mexico

h Instituto Vocacional Enrique Díaz de León, Guadalajara, Mexico

i Instituto de Prevención Social, Asunción, Paraguay

${ }^{\mathrm{j}}$ Escuela de Psicología, Universidad Dr. José Matías Delgado, San Salvador, El Salvador

${ }^{\mathrm{k}}$ Clínica de rehabilitación Las Araucarias, Buenos Aires, Argentina

${ }^{1}$ Servicio Médico Legal, Ministerio de Justicia, Santiago, Chile

${ }^{\mathrm{m}}$ Fundación Horizontes, Santa Cruz de la Sierra, Bolivia

${ }^{\mathrm{n}}$ CETYS University, Mexicali, Mexico

${ }^{\circ}$ Instituto de Neuropsicología y Demencias, Lima, Peru

${ }^{\mathrm{p}}$ Departamento de Medicina de Rehabilitación, Universidad Nacional Autónoma de Honduras, Tegucigalpa, Honduras

${ }^{\mathrm{q}}$ Departamento de psicología, Universidad del Valle de Guatemala, Guatemala City, Guatemala

${ }^{\mathrm{r}}$ Department of Psychology, Virginia Commonwealth University, Richmond, VA, USA

\begin{abstract}
.
OBJECTIVE: To generate normative data on the Symbol Digit Modalities Test (SDMT) across 11 countries in Latin America, with country-specific adjustments for gender, age, and education, where appropriate.

METHOD: The sample consisted of 3,977 healthy adults who were recruited from Argentina, Bolivia, Chile, Cuba, El Salvador, Guatemala, Honduras, Mexico, Paraguay, Peru, and, Puerto Rico. Each subject was administered the SDMT as part of a larger neuropsychological battery. A standardized five-step statistical procedure was used to generate the norms.

RESULTS: The final multiple linear regression models explained $29-56 \%$ of the variance in SDMT scores. Although there were gender differences on the SDMT in Mexico, Honduras, Paraguay, and Guatemala, none of the four countries had an effect size greater than 0.3 . As a result, gender-adjusted norms were not generated.

CONCLUSIONS: This is the first normative multicenter study conducted in Latin America to create norms for the SDMT; this study will have an impact on the future practice of neuropsychology throughout the global region.
\end{abstract}

Keywords: Normative data, Symbol Digit Modalities Test, reference values, Latin America, processing speed, attention

*Address for correspondence: Juan Carlos Arango-Lasprilla, Ph.D., IKERBASQUE Research Professor, Department of Psychology, University of Deusto, IKERBASQUE, Basque
Foundation for Science, Bilbao, Spain. Tel.: +34 $94 \quad 413 \quad 90$ 03 (Extn. 3261); Fax: +34 9441393 42. E-mail: jcarango@ deusto.es. 


\section{Introduction}

The Symbol Digit Modalities Test (SDMT) is a paper-and-pencil substitution task which is easily administered and sensitive to the presence of cerebral dysfunction (Smith, 1982). Originally published in 1973 and later revised (Smith, 1982), the SDMT is considered a measure of divided attention, complex scanning and visual tracking, perceptual and motor speed, and memory (Shum, McFarland, \& Bain, 1990; Lezak, Howieson, \& Loring, 2004).

The SDMT consists of a key with two rows, with nine stimulus symbols in the upper row and matched numbers (1-9) in the row below it. The task sequence consists of a series of symbols, each with a blank space underneath, which the subject is asked to consecutively fill with the corresponding numbers as fast as possible in 90 seconds after completing a 10-item practice trial. The score of the test is the number of correct substitutions completed within the time limit, with the maximum score of 110 (Smith, 1982). Obtaining a score under 33 on the SDMT is generally considered a clear indicator of the existence of some type of cognitive disorder (Cherbuin, Sachdev, \& Anstey, 2010). The SDMT is very similar to the Digit Symbol Coding subtest of the WAIS (Wechsler, 1981), with which it correlates very highly, suggesting they are essentially the same (Morgan \& Wheelock, 1992). However, the SDMT has the advantage of allowing for responses with a more familiar act of number writing, as well as a spoken response trial (Smith, 1982; Lezak et al., 2004). A correlation on the order of 0.80 has been reported between the standard SDMT and its oral version, as well as Wechsler Digit Symbol Coding, confirming its content and construct validity (Smith, 1991; Bowler, Sudia, Mergler, Harrison, \& Cone, 1992; Lezak et al., 2004). Test-retest reliability in a sample of young athletes ranged from 0.74 to 0.91 , and from 0.84 to 0.93 in healthy adults (Hinton-Bayre, Geffen, \& McFarland, 1997; Hinton-Bayre, Geffen, Geffen, McFarland, \& Frijs, 1999; Amodio et al., 2002; Benedict, Smerbeck, Parikh, Rodgers, Cadavid, \& Erlanger, 2012).

Due to its wide applicability and ease of administration, the SDMT has been used to study cognitive sequelae in a variety of neurological conditions, including the consequences of traumatic head injury in adults (Ponsford \& Kinsella, 1992; Hingon-Bayre, Geffen, \& McFarland, 1997; Felmingham, Baguley, \& Green, 2004), brain damage in children (Lewandowski, 1984), aging and dementia (Pfeffer et al., 1981), hepatic encephalopathy (Tarter, Hegedus, Van Thiel, Schade,
Gavaler, \& Starzl., 1984; Quero, Hartmann, Meulstee, Hop, \& Schalm, 1996), extra-pyramidal disorders (Starkstein et al., 1988; Starkstein, Bolduc, Preziosi, \& Robinson, 1989), multiple sclerosis (Benedict et al., 2002; Huijbregts, Kalkers, de Sonneville, de Groot, Reuling, \& Polman, 2004; Nocentini et al., 2006), and schizophrenia (Dickinson, Ramsey, \& Gold, 2007).

Performance on the SDMT is affected by age and educational level: scores have been shown to decline with increasing age and lower education levels (Smith, 1982; Strauss, Shermann, \& Spreen, 2006), while improved performance was noted with increasing verbal IQ scores (Nielsen, Knudsen, \& Daugbjerg, 1989; Waldmann, Dickson, Monahan, \& Kazelskis, 1992). Age-related decline in performance likely reflects changes in the speed of both motor response and information processing (Gilmore, Royer, \& Gruhn, 1983) and in memory (Joy, Kaplan, \& Fein, 2004). Some studies have also found gender effects (with women outperforming men), although this finding is not consistent, tends to diminish when controlling for handedness (Waldmann, Dickson, Monahan, \& Kazelskis, 1992; Lezak et al., 2004; Strauss et al., 2006), and appears to be of insufficient magnitude to create separate genderbased norms (Gilmore, Royer, \& Gruhn, 1983).

The SDMT normative data, while accounting for such factors as age and level of education, are lacking for adults from diverse racial/ethnic groups as well as diverse global regions (Sheridan et al., 2006). This is an important issue, considering the well-documented effect of culture on differences in performance in nearly all domains of neuropsychological functioning (O'Bryant, Humphreys, Bauer, McCaffrey, \& Hilsabeck, 2007). In recent years, a number of normative studies have taken place, including among African Americans, Blacks of Caribbean ancestry, and nonLatino Whites living in areas of the United States with large populations of Blacks (Gonzalez, Whitfield, West, Williams, Lichtenberg, \& Jackson, 2007), Italian adults (Amodio et al., 2002; Nocentini, Giordano, Di Vincenzo, Panella, \& Pasqualetti, 2006), healthy elderly Danes (Vogel, Stokholm, \& Jorgensen, 2013), and in Spain among healthy adults (Smith, 2002) and institutionalized elderly persons (Cancela, Ayan, \& Varela, 2012).

Normative data are needed to implement the SDMT correctly in Latin America. To date, only limited normative data have been produced on the SDMT in Spanish from Spain but none from Latin America. The unique educational opportunities and cultures in this region underscore the necessity of norms standard- 
ized for Latin America, as interpreting performance on the SDMT in individuals from this region using norms from other countries and languages might result in significant assessment errors. As a result, the purpose of the current study was to generate norms for the SDMT across 11 countries in Latin America with appropriate adjustments for age, gender, and formal education.

\section{Method}

\subsection{Participants}

The sample consisted of 3,977 healthy individuals who were recruited from Argentina, Bolivia, Chile, Cuba, El Salvador, Guatemala, Honduras, Mexico, Paraguay, Peru, and Puerto Rico. The participants were selected according to the following criteria: a) were between 18 to 95 years of age, b) were born and currently lived in the country where the protocol was conducted, c) spoke Spanish as their native language, d) had completed at least one year of formal education, e) were able to read and write at the time of evaluation, f) scored $\geq 23$ on the Mini-Mental State Examination (MMSE, Folstein, Folstein, \& McHugh, 1975), g) scored $\leq 4$ on the Patient Health Questionnaire-9 (PHQ-9, Kroenke, Spitzer, \& Williams, 2001), and h) scored $\geq 90$ on the Barthel Index (Mahoney \& Barthel, 1965).

Participants with self-reported neurologic or psychiatric disorders were excluded due to a potential effect on cognitive performance. Participants were volunteers from the community and signed an informed consent form. Twelve participants were excluded from the analyses, with a final sample of 3965 participants. Socio-demographic and participant characteristics for each of the countries' samples have been reported elsewhere (Guàrdia-Olmos, Peró-Cebollero, Rivera, \& Arango-Lasprilla, 2015). The multi-center study was approved by the Ethics Committee of the coordinating site, the University of Deusto, Spain.

\subsection{Instrument administration}

The SDMT test consists of converting symbols with shaped geometric figures in numbers. This substitution can be applied orally or in writing. This study used normative data for the written form. When administrating the test, confirmation that the subject is marking the answers in the order given and without skipping any lines is necessary. The subject's score is the number of correct substitutions made at an interval of 90 seconds.

\subsection{Statistical analyses}

The detailed statistical analyses used to generate the normative data for this test are described in Guàrdia-Olmos et al. (2015). In summary, the data manipulation process for each country-specific dataset involved five-steps: a) $t$ - tests for independent samples and effect sizes $(r)$ were conducted to determine gender effects. If the effect size was larger than 0.3 , gender was included in the model with gender dummy coded and female as the reference group (male $=1$ and female $=0$ ); b) A multivariable regression model was used to specify the predictive model including gender (if effect size was larger than 0.3), age as a continuous variable, and education as a dummy coded variable with 1 if the participant had $>12$ years of education and 0 if the participants had 1-12 years of education. If gender, age and/or education was not statistically significant in this multivariate model with an alpha of 0.05 , the non-significant variables were removed and the model was re-run. Then a final regression model was conducted that included age (if statistically significant in the multivariable model), dichotomized education (if statistically significant in the multivariate model), and/or gender (if effect size was greater than 0.3) $\left[\hat{y}_{i}=\beta_{0}+\left(\beta_{\text {Age }} \cdot A g e_{i}\right)+\left(\beta_{E d u c}\right.\right.$. $\left.E_{\text {duc }}\right)+\left(\beta_{\text {Gender }} \cdot\right.$ Gender $\left.\left.\left._{i}\right)\right] ; \mathrm{c}\right)$ residual scores were calculated based on this final model $\left.\left(e_{i}=y_{i}-\hat{y}_{i}\right) ; \mathrm{d}\right)$ using the $S D_{e}$ (residual) value provided by the regression model, residuals were standardized: $z=e_{i} / S D_{e}$, with $S D_{e}$ (residual)= the standard deviation of the residuals in the normative sample; and e) standardized residuals were converted to percentile values (Strauss et al., 2006). Using each country's dataset, these steps were applied to SDMT scores.

\section{Results}

Regarding the effect of gender on SDMT, the $t$-tests showed significant differences between men and women in the countries of Honduras, Mexico, and Paraguay, however, none of these three countries had an effect size larger than 0.3 . Table 1 shows the results of the gender analyses by country on SDMT scores. As shown in Table 1, the effect sizes for all countries were 
Table 1

Effect of gender in the SDMT

\begin{tabular}{|c|c|c|c|c|c|c|}
\hline Country & Gender & Mean (SD) & $t$ & $\mathrm{df}$ & Sig. (2-tailed) & $r$ \\
\hline \multirow[t]{2}{*}{ Argentina $^{\mathrm{a}}$} & Male & $43.9(10.1)$ & -0.27 & 219.2 & 0.791 & $\overline{0.018}$ \\
\hline & Female & $44.2(12.5)$ & & & & \\
\hline \multirow[t]{2}{*}{ Bolivia } & Male & $27.7(17.6)$ & -0.29 & 272 & 0.775 & 0.017 \\
\hline & Female & $28.4(18.7)$ & & & & \\
\hline \multirow[t]{2}{*}{ Chile } & Male & $34.4(19.0)$ & -0.95 & 318 & 0.341 & 0.053 \\
\hline & Female & $36.5(19.9)$ & & & & \\
\hline \multirow[t]{2}{*}{ Cuba } & Male & $33.0(12.4)$ & -0.19 & 304 & 0.853 & 0.011 \\
\hline & Female & $33.3(13.4)$ & & & & \\
\hline \multirow[t]{2}{*}{ El Salvador ${ }^{\mathrm{a}}$} & Male & $26.5(16.9)$ & 1.39 & 180.5 & 0.165 & 0.103 \\
\hline & Female & $23.7(14.0)$ & & & & \\
\hline \multirow[t]{2}{*}{ Guatemala } & Male & $34.8(16.2)$ & -1.59 & 210 & 0.113 & 0.109 \\
\hline & Female & $38.3(16.0)$ & & & & \\
\hline \multirow[t]{2}{*}{ Honduras } & Male & $29.7(14.7)$ & 3.20 & 178 & $0.002^{* *}$ & 0.233 \\
\hline & Female & $23.0(12.6)$ & & & & \\
\hline \multirow[t]{2}{*}{ Mexico } & Male & $39.2(16.2)$ & 2.78 & 1,295 & $0.006^{* *}$ & 0.077 \\
\hline & Female & $36.6(15.7)$ & & & & \\
\hline \multirow[t]{2}{*}{ Paraguaya $^{a}$} & Male & $24.2(10.4)$ & 2.39 & 176.1 & $0.018^{*}$ & 0.177 \\
\hline & Female & $21.3(8.2)$ & & & & \\
\hline \multirow[t]{2}{*}{ Peru $^{a}$} & Male & $41.5(12.2)$ & 0.23 & 206.4 & 0.818 & 0.016 \\
\hline & Female & $41.1(14.8)$ & & & & \\
\hline \multirow[t]{2}{*}{ Puerto Rico } & Male & $40.4(16.5)$ & 0.43 & 290 & 0.669 & 0.025 \\
\hline & Female & $39.7(15.1)$ & & & & \\
\hline
\end{tabular}

${ }^{a}$ Value of the $t$-test for independent groups from the different variances with the corresponding correction of Yuen-Welch of degrees of freedom. ${ }^{*} p<0.05,{ }^{* *} p<0.01$.

less than 0.3, and therefore gender was not taken into account to generate SDMT normative data for any of the countries in the study.

The final eleven SDMT multivariate linear regression models for each country are shown in Table 2 . In all countries, the SDMT score increased for those with more than 12 years of education (see Table 2) and, in all countries SDMT scores decreased in a linear fashion as a function of age. The amount of variance explained in SDMT scores ranged from $30 \%$ (in Guatemala) to $56 \%$ (in Chile).

\subsection{Normative procedure}

Norms (e.g., a percentile score) for the SDMT score test were established using the five-step procedure described above. To facilitate the understanding of the procedure to obtain the percentile associated with a score on this test, an example will be given. Suppose you need to find the percentile score for a Mexican woman, who is 25 years old and has 13 years of education. She has a score of 40 on the SDMT test. The steps to obtain the percentile for this score are: a) Check Table 1 to determine if the effect size of gender in the country of interest (Mexico) on this test and time point (SDMT) is greater than 0.3 by country. In this example, the effect size is 0.077 , which is not greater than 0.3. For Mexican on this test, gender does not influence scores to a sufficient degree to take it into account when determining the percentile. b) Find Mexico in Table 2, which provides the final regression models by country for SDMT. Use the B weights to create an equation that will allow you to obtain the predicted SDMT score. The corresponding $\mathrm{B}$ weights are multiplied by the actual age and dichotomized education scores and added to a constant in order to calculate the predicted value. In this case, the predicted SDMT score would be calculated using the equation $\left[\hat{y}_{i}=60.802+\left(-0.471 \cdot\right.\right.$ Age $\left._{i}\right)+$ (6.229 . Dichotomized Educational Level ${ }_{i}$ )] (the values have been rounded for presentation in the formula). The subscript notation $i$ indicates the person of interest. The person's age is 25 , but the education variable is not continuous in the model. Years of education is split into either 1 to 12 years (and assigned a 0) or more than 12 years (and assigned a 1) in the model. Since our hypothetical person in the example has 13 years of education, her educational level value is 1 . Thus the predicted value is $\hat{y}_{i}=60.802+(-0.471 \cdot 25)+(6.229 \cdot 1)=$ $60.802-11.778+6.229=55.25$. c) In order to calculate the residual value (indicated with an $e$ in the equation), we subtract the actual value from the predicted value we just calculated $\left(e_{i}=y_{i}-\hat{y}_{i}\right)$. In this case, it would be $e_{i}=40-55.25=-15.25$. d) Next, consult the $S D_{e}$ column in Table 2 to obtain the country- 
Table 2

Final multiple linear regression models for SDMT

\begin{tabular}{|c|c|c|c|c|c|c|c|}
\hline Country & & $\mathrm{B}$ & Std. Error & $t$ & Sig. & $\mathrm{R}^{2}$ & $S D_{e}$ (residual) \\
\hline \multirow[t]{3}{*}{ Argentina } & (Constant) & 51.557 & 1.516 & 34.013 & $<0.001$ & 0.380 & 9.294 \\
\hline & Age & -0.267 & 0.027 & -9.901 & $<0.001$ & & \\
\hline & Education & 8.855 & 1.055 & 8.395 & $<0.001$ & & \\
\hline \multirow[t]{3}{*}{ Bolivia } & (Constant) & 54.010 & 2.434 & 22.193 & $<0.001$ & 0.405 & 14.082 \\
\hline & Age & -0.489 & 0.039 & -12.460 & $<0.001$ & & \\
\hline & Education & 8.180 & 2.268 & 3.606 & $<0.001$ & & \\
\hline \multirow[t]{3}{*}{ Chile } & (Constant) & 62.742 & 2.421 & 25.917 & $<0.001$ & 0.557 & 12.995 \\
\hline & Age & -0.563 & 0.039 & -14.482 & $<0.001$ & & \\
\hline & Education & 15.798 & 1.767 & 8.942 & $<0.001$ & & \\
\hline \multirow[t]{3}{*}{ Cuba } & (Constant) & 49.802 & 1.726 & 28.852 & $<0.001$ & 0.379 & 10.163 \\
\hline & Age & -0.351 & 0.030 & -11.830 & $<0.001$ & & \\
\hline & Education & 8.171 & 1.377 & 5.933 & $<0.001$ & & \\
\hline \multirow[t]{3}{*}{ El Salvador } & (Constant) & 40.460 & 2.040 & 19.836 & $<0.001$ & 0.483 & 10.956 \\
\hline & Age & -0.348 & 0.033 & -10.437 & $<0.001$ & & \\
\hline & Education & 17.849 & 1.689 & 10.569 & $<0.001$ & & \\
\hline \multirow[t]{3}{*}{ Guatemala } & (Constant) & 45.901 & 3.117 & 14.726 & $<0.001$ & 0.299 & 13.544 \\
\hline & Age & -0.282 & 0.054 & -5.188 & $<0.001$ & & \\
\hline & Education & 15.014 & 1.924 & 7.804 & $<0.001$ & & \\
\hline \multirow[t]{3}{*}{ Honduras } & (Constant) & 37.853 & 2.208 & 17.147 & $<0.001$ & 0.486 & 9.874 \\
\hline & Age & -0.331 & 0.041 & -8.159 & $<0.001$ & & \\
\hline & Education & 14.479 & 1.757 & 8.241 & $<0.001$ & & \\
\hline \multirow{3}{*}{ Mexico } & (Constant) & 60.802 & 0.972 & 62.534 & $<0.001$ & 0.430 & 12.012 \\
\hline & Age & -0.471 & 0.016 & -28.592 & $<0.001$ & & \\
\hline & Education & 6.229 & 0.807 & 7.719 & $<0.001$ & & \\
\hline \multirow[t]{3}{*}{ Paraguay } & (Constant) & 32.522 & 1.812 & 17.950 & $<0.001$ & 0.441 & 6.866 \\
\hline & Age & -0.226 & 0.031 & -7.187 & $<0.001$ & & \\
\hline & Education & 10.241 & 1.211 & 8.456 & $<0.001$ & & \\
\hline \multirow[t]{3}{*}{ Peru } & (Constant) & 52.694 & 1.787 & 29.491 & $<0.001$ & 0.534 & 9.486 \\
\hline & Age & -0.404 & 0.030 & -13.377 & $<0.001$ & & \\
\hline & Education & 9.400 & 1.295 & 7.261 & $<0.001$ & & \\
\hline \multirow[t]{3}{*}{ Puerto Rico } & (Constant) & 64.815 & 2.255 & 28.739 & $<0.001$ & 0.473 & 11.380 \\
\hline & Age & -0.534 & 0.037 & -14.263 & $<0.001$ & & \\
\hline & Education & 5.010 & 1.387 & 3.611 & $<0.001$ & & \\
\hline
\end{tabular}

specific $S D_{e}$ (residual) value. For Mexico it is 12.012 . Using this value, we can transform the residual value to a standardized $z$ score using the equation $\left(e_{i} / S D_{e}\right)$. In this case, we have $(-15.25) / 12.012=-1.269$. This is the standardized $z$ score for a Mexican woman aged 25 and 13 years of education and a score of 40 on the SDMT test. e) The last step is to use look-up the tables in the statistical reference books (e.g. Strauss et al., 2006) or use a trusted online calculator like the one available at http://www.measuringu.com/pcalcz.php. In the online calculator, you would enter the $z$ score and choose a one-sided test and note the percent of area after hitting the submit button. In this case, the probability of -1.269 corresponds to the 10 th percentile.

\subsection{User-friendly normative data tables}

The five-step normative procedures explained above can provide more individualized norms. However, this method can be prone to human error due to the number of required computations. To enhance user-friendliness, the authors have completed these steps for a range of raw scores based on small age range groupings (see Guàrdia-Olmos et al., 2015) and created tables so that clinicians can more easily use to obtain a percentile range associated with a given raw score on this test. These tables are available by country and type of test (SDMT) in the Appendix. In order to obtain an approximate percentile for the above example (converting a raw score of 40 for a Mexican women who is 25 years old and has 13 years of education) using the simplified normative tables provided, the following steps are recommended. (1) First, identify the appropriate table ensuring the specific country and test. In this case, the table for SDMT scores for Mexico can be found in Table A8. (2) Note if the title of the table indicates that it is only to be used for one specific gender. In this case, gender is not specified. Thus Table A8 is used for both males and females. (3) Next, the table is divided based on educational level ( 1 to 12 vs. more than 12 years of 
education). Since this woman has 13 years of education, she falls into the more than 12 years of education category. These data can be found in the top section of the table. (4) Determine the age range most appropriate for the individual. In this case, 25 falls into the column 23-27 years of age. (5) Read down the age range column to find the approximate location of the raw score the person obtained on the text. Reading down the 23-27 column, the score of 40 obtained by this Mexican woman corresponds to an approximate percentile of 10 .

\section{Discussion}

The purpose of the current study was to generate normative data on the SDMT across 11 countries in Latin America, with country-specific adjustments for gender, age, and education, where appropriate. The final multiple linear regression models explained between $29-55 \%$ of the variance in the SDMT scores. Although men outperformed women on the SDMT in three of the 11 countries (Mexico, Honduras, and Paraguay), women outperformed men in Guatemala. Nonetheless, all gender effect sizes were small, and therefore genderadjusted norms were not generated. These findings are generally in line with the literature, as previously reported gender effects have tended to be inconsistent and to diminish when controlling for handedness (Waldmann et al., 1992; Lezak et al., 2004; Strauss et al., 2006). Previous studies have similarly found gender differences to be too small to create separate gender norms (Gilmore, Royer, \& Gruhn, 1983). In light of the previous literature, the current results suggest that gender should not be taken into account in calculating participants' percentiles for the SDMT in in Latin America when using the current norms.

The SDMT scores increased linearly as a function of education in all countries. These findings were consistent within the current study, as well as with previous studies showing that performance on the SDMT increases with education level (Smith, 1982; Strauss et al., 2006). Because of potentially major differences in the quality of education across countries in Latin America, it is very important to use the education-adjusted norms generated for a single country when administering the SDMT to individuals from that country.

Age was inversely associated with SDMT scores in all countries, and as a result, age-adjusted norms were calculated for each country. These findings are in line with the previous literature showing SDMT scores to decrease with advancing age (Smith, 1982; Strauss,
Shermann, \& Spreen, 2006), as age-related decline in SDMT performance likely reflects changes in the speed of motor response and information processing (Gilmore et al., 1983) and in memory (Joy, Kaplan, \& Fein, 2004). As with education, it is important that neuropsychologists in Latin America use the age-adjusted norms for their specific country.

\subsection{Limitations and future directions}

This study has several limitations, and as a result directions for future research. First, this study was subject to a number of sampling limitations. Participants had Spanish as their primary language, and SDMT performance could differ among people with various languages, bilingualism, or local dialects such as Quechua or Guaraní. A ripe area for future research is bilingualism and SDMT performance. Also, the data were collected in various regions of the countries in this study, as opposed to nationally. Although this study was the largest neuropsychological normative study in the history of Latin America, or of any global region, it is only a first step in conducting more rigorous, larger studies with nationally representative samples. Although many participants had fewer than 12 years of education, those who were unable to read or write were excluded; thus, the SDMT norms cannot generalize to illiterate populations. Similarly, all participants in the current study were healthy adults, so future studies need to include populations with neurological conditions, as well as children. Future research may also assess participants' bilingualism, which was not controlled for or examined.

Second, clinicians should be cautious about using the norms of the SDMT from the current study in countries in Latin America beyond the 11 countries from which data were collected. In the future, it is important to establish SDMT norms in other countries such as Ecuador, Uruguay, Venezuela, and Panama. Nonetheless, the current SDMT norms may be more accurate in these countries than other norms from Spain or Englishspeaking countries that may currently be in use; future research should nonetheless investigate the generalizability of the current norms.

Third, despite the SDMT's commonness in Latin America, many other assessments are also common and need to be normed in the same manner. It is important that future research examine the psychometric properties of common neuropsychological instruments in Latin America, even at the individual item level. Research should also examine whether 
neuropsychological assessments have good ecological validity, or develop instruments in those cultures with higher ecological validity. The SDMT was created in a Western culture that may be different from the various cultures in Latin America. Future research should develop more culturally sensitive assessments bound in local cultures, not solely translate and norm tests from other cultures and countries.

Despite these limitations and although previous studies have produced Spanish-language norms for the SDMT in Spain with healthy adults (Smith, 2002), the current study was the first to generate SDMT norms across 11 countries in Latin America with nearly 4,000 participants. This study was the largest and most comprehensive SDMT normative study to date in any global region, and its norms have the potential to affect the standard of neuropsychological assessment with the SDMT in Latin America unlike any study before it.

\section{References}

Amodio, P., Wenin, H., del Piccolo, F., Mapelli, D., Montagnese, S., Pellegrini, A., Musto, C., Gatta, A., \& Umilta, C. (2002). Variability of Trail Making Test, Symbol Digit Test and Line Trait Test in normal people. A normative study taking into account agedependent decline and sociobiological variables. Aging Clinical and Experimental Research, 14(2), 117-131.

Benedict, R. H., Smerbeck, A., Parikh, R., Rodgers, J., Cadavid, D., \& Erlanger, D. (2012). Reliability and equivalence of alternate forms for the Symbol Digit Modalities Test: Implications for multiple sclerosis clinical trials. Multiple Sclerosis Journal, 18(9), 13201325.

Benedict, R., Fischer, J., Archibald, C., Arnett, P., Beatty, W., Bobholz, J., Chelune, G., Fisk, J., Langdon, D., Caruso, L., Foley, F., LaRocca, N., Vowels, L., Weinstein, A., DeLuca, J., Rao, S., \& Munschauer, F. (2002). Minimal neuropsychological assessment of MS patients: A consensus approach. The Clinical Neuropsychologist, 16(3), 381-397.

Bowler, R., Sudia, S., Mergler, D., Harrison, R., \& Cone, J. (1992). Comparison of digit symbol and symbol digit modalities tests for assessing neurotoxic exposure. The Clinical Neuropsychologist, 6(1), 103-104.

Cancela, J., Ayan, C., \& Varela, S. (2012). "Symbol Digit Modalities Test" normative values for Spanish home care residents: A pilot study. Actas Españolas de Psiquiatria, 40(6), 299-303.

Cherbuin, N., Sachdev, P., \& Anstey, K. (2010). Neuropsychological predictors of transition from healthy cognitive aging to mild cognitive impairment: The PATH Through Life Study. American Journal of Geriatric Psychiatry, 18(8), 723-33.

Dickinson, D., Ramsey, M., \& Gold, J. (2007). Overlooking the obvious. A meta-analytic comparison of digit symbol coding tasks and other cognitive measures in schizophrenia. Archives of General Psychiatry, 64(5), 532-542.

Felmingham, K., Baguley, I., \& Green, A. (2004). Effects of diffuse axonal injury on speed of information processing following severe traumatic brain injury. Neuropsychology, 18(3), 564-571.

Folstein, M. F., Folstein, S. E., \& McHugh, P. R. (1975). "Minimental state": A practical method for grading the cognitive state of patients for the clinician. Journal of Psychiatric Research, 12(3), 189-198.

Gilmore, G. C., Royer, F. L., \& Gruhn, J. J. (1983). Age differences in symbol-digit substitution task performance. Journal of Clinical Psychology, 39(1), 114-124.

González, H. M., Whitfield, K. E., West, B. T., Williams, D. R., Lichtenberg, P. A., \& Jackson, J. S. (2007). Modified-symbol digit modalities test for African Americans, Caribbean Black Americans, and non-Latino Whites: Nationally representative normative data from the National Survey of American Life. Archives of Clinical Neuropsychology, 22(5), 605-13.

Guàrdia-Olmos, J., Peró-Cebollero, M., Rivera, D., \& ArangoLasprilla, J.C. (2015). Methodology for the development of normative data for ten Spanish-language neuropsychological tests in eleven Latin American countries. NeuroRehabilitation, 37, 493-499.

Hinton-Bayre, A. D., Geffen, G. M., Geffen, L. B., McFarland, K. A., \& Frijs, P. (1999). Concussion in contact sports: Reliable change indices of impairment and recovery. Journal of Clinical and Experimental Neuropsychology, 21(1), 70-86.

Hinton-Bayre, A., Geffen, G., \& McFarland, K. (1997). Mild head injury and speed of information processing: A prospective study of professional rugby league players. Journal of Clinical and Experimental Neuropsychology, 19(2), 275-289.

Huijbregts, S., Kalkers, N., de Sonneville, L., de Groot, V., Reuling, I., \& Polman, C. (2004). Differences in cognitive impairment of relapsing remitting, secondary, and primary progressive MS. Neurology, 63(2), 335-339.

Joy, S., Kaplan, E., \& Fein, D. (2004). Speed and memory in the WAIS-III Digit Symbol-Coding subtest across the adult lifespan. Archives of Clinical Neuropsychology, 19, 759-767.

Kroenke, K., Spitzer, R. L., \& Williams, J. B. (2001). The PHQ-9. Journal of General Internal Medicine, 16(9), 606-613.

Lewandowski, L. (1984). The Symbol Digit Modalities Test: A screening instrument for brain damaged children. Perceptual \& Motor Skills, 59(2), 615-618.

Lezak, M., Howieson, D., \& Loring, D. (2004). Neuropsychological Assessment (4th ed.). New York: Oxford University Press.

Mahoney, F. I., \& Barthel, D. (1965). Functional evaluation: The Barthel Index. Maryland State Medical Journal, 14, 56-61.

Morgan, S., \& Wheelock, J. (1992). Digit symbol and symbol digit modalities tests: Are they directly interchangeable? Neuropsychology, 6(4), 327-330.

Nielsen, H., Knudsen, L., \& Daugbjerg, O. (1989). Normative data for eight neuropsychological tests based on a Danish sample. Scandinavian Journal of Psychology, 30(1), 37-45.

Nocentini, U., Giordano, A., Di Vincenzo, S., Panella, M., \& Pasqualetti, P. (2006). The Symbol Digit Modalities Test - Oral versión: Italian normative data. Functional Neurology, 21(2), 93-96.

Nocentini, U., Pasqualetti, P., Bonavita, S., Buccafusca, M., De Caro, M., Farina, D., Girlanda, P., Le Pira, F., Lugaresi, A., Quattrone, A., Reggio, A., Salemi, G., Savettieri, G., Tedeschi, G., Trojano, M., Valentino, P., \& Caltagirone, C. (2006). Cognitive dysfunction in patients with relapsing-remitting multiple sclerosis. Multiple Sclerosis, 12(1), 77-87. 
O’Bryant, S., Hemphreys, J., Bauer, L., McCaffrey, R., \& Hilsabeck, R. (2007). The influence of ethnicity on symbol digit modalities test performance: An analysis of a multi-ethnic college and hepatitis C patient sample. Applied Neuropsychology, 14(3), 183-188.

Pfeffer RI, Kurosaki TT, Harrah, C., Chance, J., Bates, D., Detels, R., Filos, S., \& Butzke, C. (1981). A survey diagnostic tool for senile dementia. American Journal of Epidemiology, 114(4), 515-527.

Ponsford, J., \& Kinsella, G. (1992). Attentional deficits following closed head injury. Journal of Clinical and Experimental Neuropsychology, 14(5), 822-838.

Quero, J., Hartmann, I., Meulstee, J., Hop, W., \& Schalm, S. (1996). The diagnosis of subclinical hepatic encephalopathy in patients with cirrhosis using neuropsychological tests and automated electroencephalogram analysis. Hepatology, 24(3), 556-560.

Sheridan, L., Fitzgerald, H., Adams, K., Nigg, J., Martel, M., Puttler, L., Wong, M., \& Zucker, R. (2006). Normative symbol digit modalities test performance in a community-based sample. Archives of Clinical Neuropsychology, 21(1), 23-28.

Shum, D., McFarland, K., \& Bain, J. (1990). Construct validity of eight tests of attention: Comparison of normal and closed head injured samples. Clinical Neuropsychology, 4(2), 151-62.

Smith, A. (1982). Symbol Digit Modalities Test (SDMT). Manual (revised). Los Angeles: Western Psychological Services.

Smith, A. (1991). Symbol Digit Modalities Test. Los Angeles, CA: Western Psychological Services.

Smith, A. (2002). Test de símbolos y dígitos (Symbol and Digit Test). Madrid: TEA Ediciones S.A.
Starkstein, S., Bolduc, P., Preziosi, T., \& Robinson, R. (1989). Cognitive impairments in different stages of Parkinson's disease. Journal of Neuropsychiatry \& Clinical Neuroscience, 1, 243-248.

Starkstein, S. E., Brandt, J., Folstein, S., Strauss, M., Berthier, M., Pearlson, G., Wong, D., McDonnell, A., \& Folstein, M. (1988). Neuropsychological and neuroradiological correlates in Huntington's disease. Journal of Neurology Neurosurgery \& Psychiatry, 51(10), 1259-1263.

Strauss, E., Sherman, E., \& Spreen, O. (2006). A compendium of neuropsychological tests: Administration, norms, and commentary (3rd ed.). New York: Oxford University Press.

Tarter, R., Hegedus, A., Van Thiel, D., Schade, R., Gavaler, J., \& Starzl, T. (1984). Nonalcoholic cirrhosis associated with neuropsychological dysfunction in the absence of overt evidence of hepatic encephalopathy. Gastroenterology, 86(6), 1421-1427.

Vogel, A., Stokholm, J., \& Jorgensen, K. (2013). Performances on Symbol Digit Modalities Test, Color Trails Test, and modified Stroop test in a healthy, elderly Danish sample. Aging, Neuropsychology, and Cognition, 20(3), 370-382.

Waldmann, B. W., Dickson, A. L., Monahan, M. C., \& Kazelskis, R. (1992). The relationship between intellectual ability and adult performance on the Trail Making Test and the Symbol Digit Modalities Test. Journal of Clinical Psychology, 48(3), 360-363.

Wechsler, D. (1981). The Wechsler Adult Intelligence Scale-Revised. New York: The Psychological Corporation. 
Appendix

Table A1

Normative data for the SDMT stratified by age and education levels for ARGENTINA

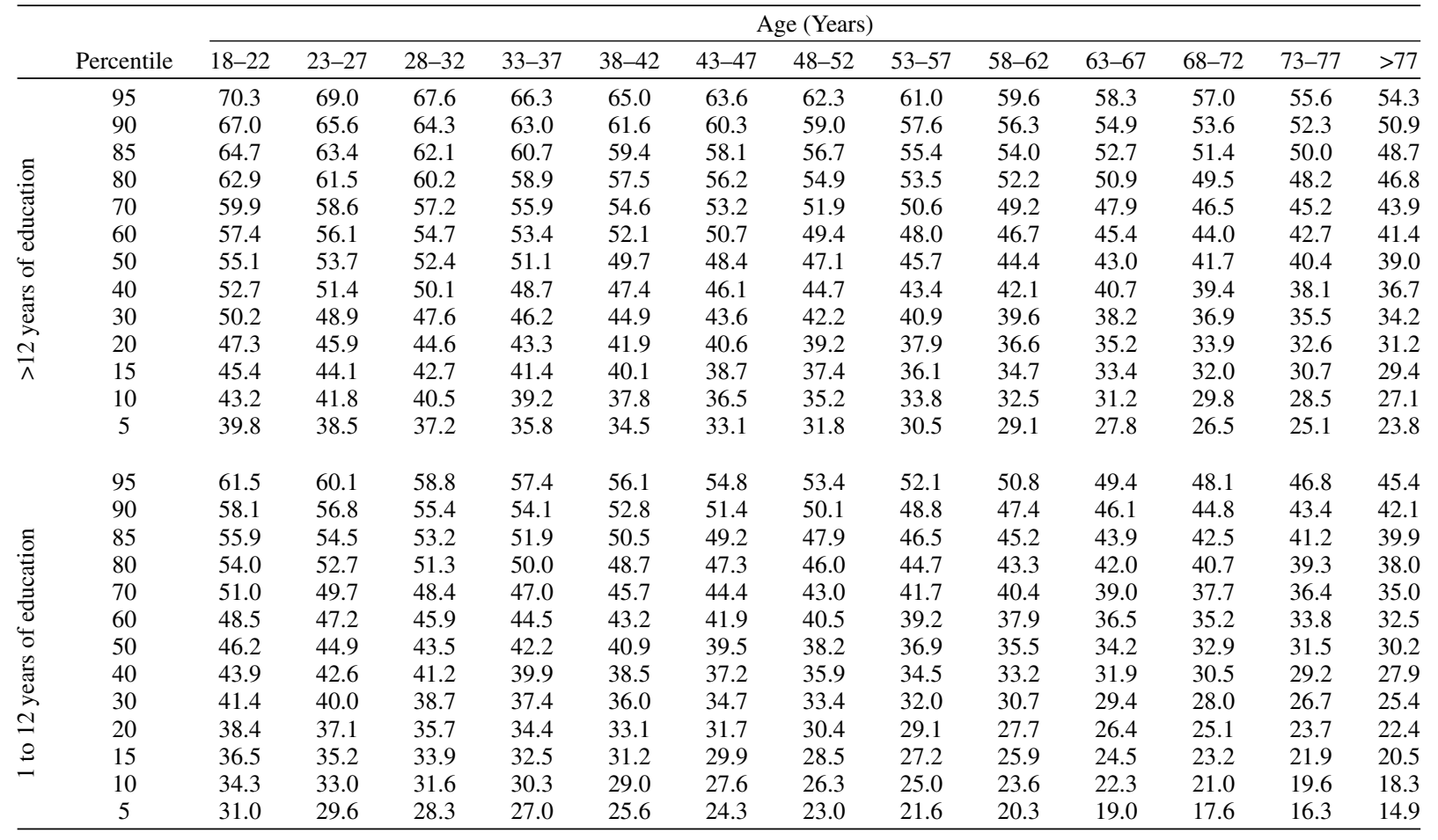

Table A2

Normative data for the SDMT stratified by age and education levels for BOLIVIA

\begin{tabular}{|c|c|c|c|c|c|c|c|c|c|c|c|c|c|c|}
\hline & \multirow[b]{2}{*}{ Percentile } & \multicolumn{13}{|c|}{ Age (Years) } \\
\hline & & $18-22$ & $23-27$ & $28-32$ & $33-37$ & $38-42$ & $43-47$ & $48-52$ & $53-57$ & $58-62$ & $63-67$ & $68-72$ & $73-77$ & $>77$ \\
\hline \multirow{13}{*}{ 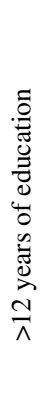 } & 95 & 75.5 & 73.1 & 70.6 & 68.2 & 65.7 & 63.3 & 60.8 & 58.4 & 55.9 & 53.5 & 51.1 & 48.6 & 46.2 \\
\hline & 90 & 70.4 & 68.0 & 65.5 & 63.1 & 60.7 & 58.2 & 55.8 & 53.3 & 50.9 & 48.4 & 46.0 & 43.5 & 41.1 \\
\hline & 85 & 67.1 & 64.6 & 62.2 & 59.7 & 57.3 & 54.8 & 52.4 & 49.9 & 47.5 & 45.0 & 42.6 & 40.2 & 37.7 \\
\hline & 80 & 64.2 & 61.8 & 59.3 & 56.9 & 54.5 & 52.0 & 49.6 & 47.1 & 44.7 & 42.2 & 39.8 & 37.3 & 34.9 \\
\hline & 70 & 59.7 & 57.3 & 54.8 & 52.4 & 50.0 & 47.5 & 45.1 & 42.6 & 40.2 & 37.7 & 35.3 & 32.8 & 30.4 \\
\hline & 60 & 55.9 & 53.5 & 51.0 & 48.6 & 46.1 & 43.7 & 41.3 & 38.8 & 36.4 & 33.9 & 31.5 & 29.0 & 26.6 \\
\hline & 50 & 52.4 & 50.0 & 47.5 & 45.1 & 42.6 & 40.2 & 37.7 & 35.3 & 32.8 & 30.4 & 28.0 & 25.5 & 23.1 \\
\hline & 40 & 48.9 & 46.4 & 44.0 & 41.6 & 39.1 & 36.7 & 34.2 & 31.8 & 29.3 & 26.9 & 24.4 & 22.0 & 19.5 \\
\hline & 30 & 45.1 & 42.6 & 40.2 & 37.8 & 35.3 & 32.9 & 30.4 & 28.0 & 25.5 & 23.1 & 20.6 & 18.2 & 15.7 \\
\hline & 20 & 40.6 & 38.1 & 35.7 & 33.2 & 30.8 & 28.4 & 25.9 & 23.5 & 21.0 & 18.6 & 16.1 & 13.7 & 11.2 \\
\hline & 15 & 37.8 & 35.3 & 32.9 & 30.4 & 28.0 & 25.5 & 23.1 & 20.6 & 18.2 & 15.8 & 13.3 & 10.9 & 8.4 \\
\hline & 10 & 34.4 & 31.9 & 29.5 & 27.0 & 24.6 & 22.2 & 19.7 & 17.3 & 14.8 & 12.4 & 9.9 & 7.5 & 5.0 \\
\hline & 5 & 29.3 & 26.9 & 24.4 & 22.0 & 19.5 & 17.1 & 14.6 & 12.2 & 9.8 & 7.3 & 4.9 & 2.4 & 0.0 \\
\hline \multirow{13}{*}{ 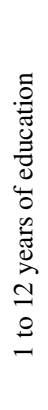 } & 95 & 67.3 & 64.9 & 62.4 & 60.0 & 57.5 & 55.1 & 52.7 & 50.2 & 47.8 & 45.3 & 42.9 & 40.4 & 38.0 \\
\hline & 90 & 62.3 & 59.8 & 57.4 & 54.9 & 52.5 & 50.0 & 47.6 & 45.1 & 42.7 & 40.2 & 37.8 & 35.4 & 32.9 \\
\hline & 85 & 58.9 & 56.4 & 54.0 & 51.5 & 49.1 & 46.6 & 44.2 & 41.8 & 39.3 & 36.9 & 34.4 & 32.0 & 29.5 \\
\hline & 80 & 56.1 & 53.6 & 51.2 & 48.7 & 46.3 & 43.8 & 41.4 & 38.9 & 36.5 & 34.1 & 31.6 & 29.2 & 26.7 \\
\hline & 70 & 51.6 & 49.1 & 46.7 & 44.2 & 41.8 & 39.3 & 36.9 & 34.4 & 32.0 & 29.5 & 27.1 & 24.7 & 22.2 \\
\hline & 60 & 47.7 & 45.3 & 42.9 & 40.4 & 38.0 & 35.5 & 33.1 & 30.6 & 28.2 & 25.7 & 23.3 & 20.9 & 18.4 \\
\hline & 50 & 44.2 & 41.8 & 39.3 & 36.9 & 34.4 & 32.0 & 29.6 & 27.1 & 24.7 & 22.2 & 19.8 & 17.3 & 14.9 \\
\hline & 40 & 40.7 & 38.3 & 35.8 & 33.4 & 30.9 & 28.5 & 26.0 & 23.6 & 21.1 & 18.7 & 16.3 & 13.8 & 11.4 \\
\hline & 30 & 36.9 & 34.5 & 32.0 & 29.6 & 27.1 & 24.7 & 22.2 & 19.8 & 17.3 & 14.9 & 12.5 & 10.0 & 7.6 \\
\hline & 20 & 32.4 & 30.0 & 27.5 & 25.1 & 22.6 & 20.2 & 17.7 & 15.3 & 12.8 & 10.4 & 7.9 & 5.5 & 3.1 \\
\hline & 15 & 29.6 & 27.1 & 24.7 & 22.2 & 19.8 & 17.4 & 14.9 & 12.5 & 10.0 & 7.6 & 5.1 & 2.7 & 0.2 \\
\hline & 10 & 26.2 & 23.8 & 21.3 & 18.9 & 16.4 & 14.0 & 11.5 & 9.1 & 6.6 & 4.2 & 1.8 & - & - \\
\hline & 5 & 21.1 & 18.7 & 16.2 & 13.8 & 11.4 & 8.9 & 6.5 & 4.0 & 1.6 & - & - & - & - \\
\hline
\end{tabular}


Table A3

Normative data for the SDMT stratified by age and education levels for CHILE

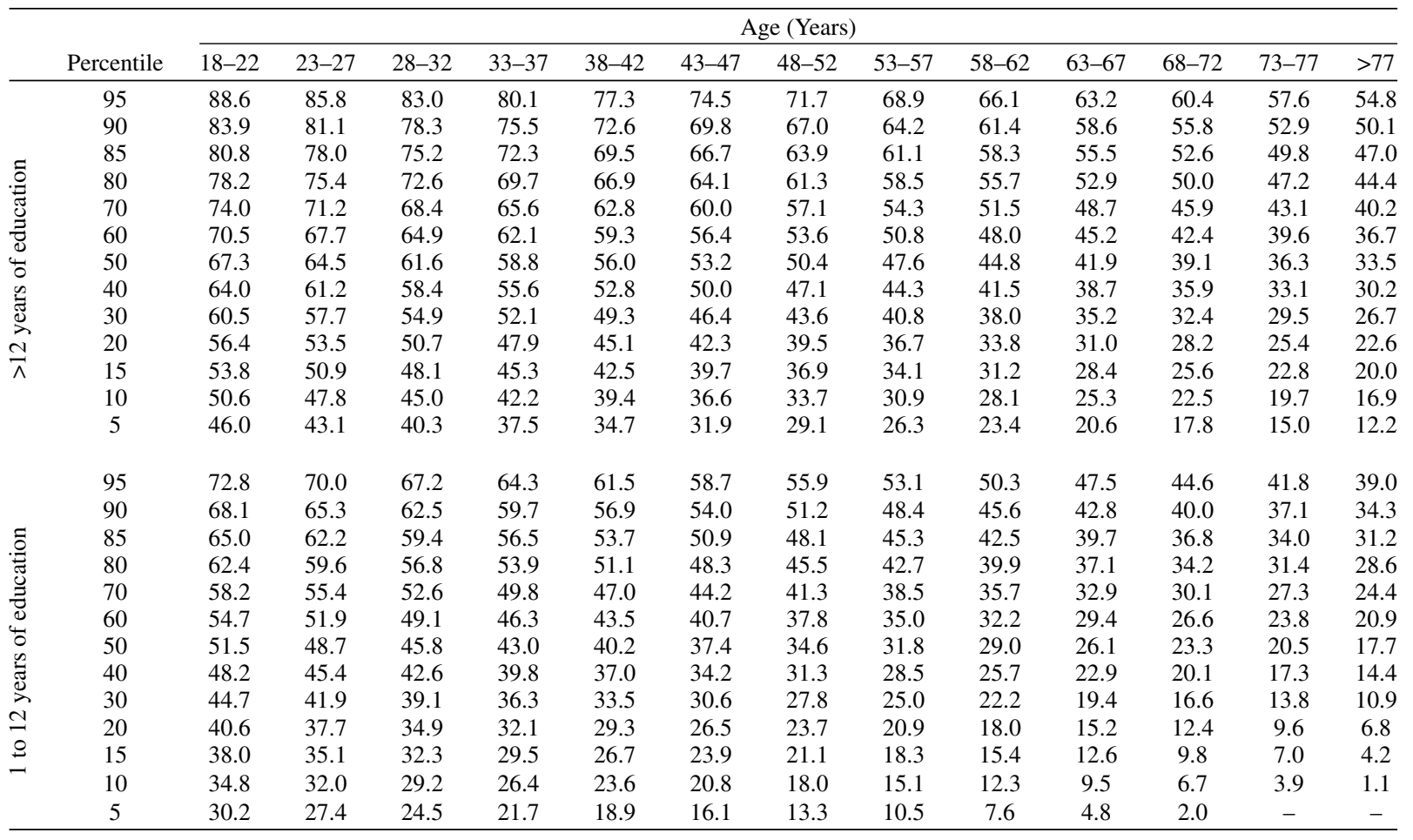

Table A4

Normative data for the SDMT stratified by age and education levels for CUBA

\begin{tabular}{|c|c|c|c|c|c|c|c|c|c|c|c|c|c|c|}
\hline \multirow{2}{*}{\multicolumn{2}{|c|}{ rcentile }} & \multicolumn{13}{|c|}{ Age (Years) } \\
\hline & & $18-22$ & $23-27$ & $28-32$ & $33-37$ & $38-42$ & $43-47$ & $48-52$ & $53-57$ & $58-62$ & $63-67$ & $68-72$ & $73-77$ & $>77$ \\
\hline \multirow{13}{*}{ 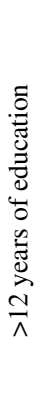 } & 95 & 67.6 & 65.9 & 64.1 & 62.4 & 60.6 & 58.9 & 57.1 & 55.4 & 53.6 & 51.8 & 50.1 & 48.3 & 46.6 \\
\hline & 90 & 64.0 & 62.2 & 60.5 & 58.7 & 57.0 & 55.2 & 53.4 & 51.7 & 49.9 & 48.2 & 46.4 & 44.7 & 42.9 \\
\hline & 85 & 61.5 & 59.8 & 58.0 & 56.3 & 54.5 & 52.8 & 51.0 & 49.3 & 47.5 & 45.7 & 44.0 & 42.2 & 40.5 \\
\hline & 80 & 59.5 & 57.7 & 56.0 & 54.2 & 52.5 & 50.7 & 49.0 & 47.2 & 45.5 & 43.7 & 42.0 & 40.2 & 38.5 \\
\hline & 70 & 56.2 & 54.5 & 52.7 & 51.0 & 49.2 & 47.5 & 45.7 & 44.0 & 42.2 & 40.5 & 38.7 & 37.0 & 35.2 \\
\hline & 60 & 53.5 & 51.7 & 50.0 & 48.2 & 46.5 & 44.7 & 43.0 & 41.2 & 39.5 & 37.7 & 36.0 & 34.2 & 32.5 \\
\hline & 50 & 51.0 & 49.2 & 47.5 & 45.7 & 43.9 & 42.2 & 40.4 & 38.7 & 36.9 & 35.2 & 33.4 & 31.7 & 29.9 \\
\hline & 40 & 48.4 & 46.7 & 44.9 & 43.2 & 41.4 & 39.7 & 37.9 & 36.1 & 34.4 & 32.6 & 30.9 & 29.1 & 27.4 \\
\hline & 30 & 45.7 & 43.9 & 42.2 & 40.4 & 38.7 & 36.9 & 35.2 & 33.4 & 31.6 & 29.9 & 28.1 & 26.4 & 24.6 \\
\hline & 20 & 42.4 & 40.7 & 38.9 & 37.2 & 35.4 & 33.7 & 31.9 & 30.1 & 28.4 & 26.6 & 24.9 & 23.1 & 21.4 \\
\hline & 15 & 40.4 & 38.6 & 36.9 & 35.1 & 33.4 & 31.6 & 29.9 & 28.1 & 26.4 & 24.6 & 22.9 & 21.1 & 19.3 \\
\hline & 10 & 38.0 & 36.2 & 34.4 & 32.7 & 30.9 & 29.2 & 27.4 & 25.7 & 23.9 & 22.2 & 20.4 & 18.7 & 16.9 \\
\hline & 5 & 34.3 & 32.5 & 30.8 & 29.0 & 27.3 & 25.5 & 23.8 & 22.0 & 20.3 & 18.5 & 16.8 & 15.0 & 13.2 \\
\hline \multirow{13}{*}{ 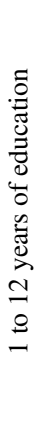 } & 95 & 59.5 & 57.7 & 55.9 & 54.2 & 52.4 & 50.7 & 48.9 & 47.2 & 45.4 & 43.7 & 41.9 & 40.2 & 38.4 \\
\hline & 90 & 55.8 & 54.0 & 52.3 & 50.5 & 48.8 & 47.0 & 45.3 & 43.5 & 41.8 & 40.0 & 38.3 & 36.5 & 34.8 \\
\hline & 85 & 53.4 & 51.6 & 49.8 & 48.1 & 46.3 & 44.6 & 42.8 & 41.1 & 39.3 & 37.6 & 35.8 & 34.1 & 32.3 \\
\hline & 80 & 51.3 & 49.6 & 47.8 & 46.1 & 44.3 & 42.6 & 40.8 & 39.0 & 37.3 & 35.5 & 33.8 & 32.0 & 30.3 \\
\hline & 70 & 48.1 & 46.3 & 44.6 & 42.8 & 41.1 & 39.3 & 37.6 & 35.8 & 34.0 & 32.3 & 30.5 & 28.8 & 27.0 \\
\hline & 60 & 45.3 & 43.6 & 41.8 & 40.1 & 38.3 & 36.6 & 34.8 & 33.1 & 31.3 & 29.5 & 27.8 & 26.0 & 24.3 \\
\hline & 50 & 42.8 & 41.0 & 39.3 & 37.5 & 35.8 & 34.0 & 32.3 & 30.5 & 28.8 & 27.0 & 25.3 & 23.5 & 21.7 \\
\hline & 40 & 40.2 & 38.5 & 36.7 & 35.0 & 33.2 & 31.5 & 29.7 & 28.0 & 26.2 & 24.5 & 22.7 & 21.0 & 19.2 \\
\hline & 30 & 37.5 & 35.7 & 34.0 & 32.2 & 30.5 & 28.7 & 27.0 & 25.2 & 23.5 & 21.7 & 20.0 & 18.2 & 16.5 \\
\hline & 20 & 34.3 & 32.5 & 30.7 & 29.0 & 27.2 & 25.5 & 23.7 & 22.0 & 20.2 & 18.5 & 16.7 & 15.0 & 13.2 \\
\hline & 15 & 32.2 & 30.5 & 28.7 & 27.0 & 25.2 & 23.5 & 21.7 & 19.9 & 18.2 & 16.4 & 14.7 & 12.9 & 11.2 \\
\hline & 10 & 29.8 & 28.0 & 26.3 & 24.5 & 22.8 & 21.0 & 19.3 & 17.5 & 15.8 & 14.0 & 12.2 & 10.5 & 8.7 \\
\hline & 5 & 26.1 & 24.4 & 22.6 & 20.9 & 19.1 & 17.4 & 15.6 & 13.8 & 12.1 & 10.3 & 8.6 & 6.8 & 5.1 \\
\hline
\end{tabular}


Table A5

Normative data for the SDMT stratified by age and education levels for EL SALVADOR

\begin{tabular}{|c|c|c|c|c|c|c|c|c|c|c|c|c|c|c|}
\hline & \multirow[b]{2}{*}{ Percentile } & \multicolumn{13}{|c|}{ Age (Years) } \\
\hline & & $18-22$ & $23-27$ & $28-32$ & $33-37$ & $38-42$ & $43-47$ & $48-52$ & $53-57$ & $58-62$ & $63-67$ & $68-72$ & $73-77$ & $>77$ \\
\hline \multirow{13}{*}{ 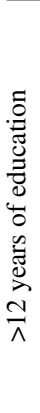 } & 95 & 69.3 & 67.6 & 65.8 & 64.1 & 62.4 & 60.6 & 58.9 & 57.2 & 55.4 & 53.7 & 51.9 & 50.2 & 48.5 \\
\hline & 90 & 65.4 & 63.6 & 61.9 & 60.2 & 58.4 & 56.7 & 55.0 & 53.2 & 51.5 & 49.7 & 48.0 & 46.3 & 44.5 \\
\hline & 85 & 62.8 & 61.0 & 59.3 & 57.5 & 55.8 & 54.1 & 52.3 & 50.6 & 48.8 & 47.1 & 45.4 & 43.6 & 41.9 \\
\hline & 80 & 60.6 & 58.8 & 57.1 & 55.3 & 53.6 & 51.9 & 50.1 & 48.4 & 46.7 & 44.9 & 43.2 & 41.4 & 39.7 \\
\hline & 70 & 57.1 & 55.3 & 53.6 & 51.8 & 50.1 & 48.4 & 46.6 & 44.9 & 43.2 & 41.4 & 39.7 & 37.9 & 36.2 \\
\hline & 60 & 54.1 & 52.4 & 50.6 & 48.9 & 47.1 & 45.4 & 43.7 & 41.9 & 40.2 & 38.5 & 36.7 & 35.0 & 33.2 \\
\hline & 50 & 51.4 & 49.6 & 47.9 & 46.1 & 44.4 & 42.7 & 40.9 & 39.2 & 37.5 & 35.7 & 34.0 & 32.2 & 30.5 \\
\hline & 40 & 48.6 & 46.9 & 45.1 & 43.4 & 41.7 & 39.9 & 38.2 & 36.5 & 34.7 & 33.0 & 31.2 & 29.5 & 27.8 \\
\hline & 30 & 45.7 & 43.9 & 42.2 & 40.4 & 38.7 & 37.0 & 35.2 & 33.5 & 31.8 & 30.0 & 28.3 & 26.5 & 24.8 \\
\hline & 20 & 42.2 & 40.4 & 38.7 & 36.9 & 35.2 & 33.5 & 31.7 & 30.0 & 28.3 & 26.5 & 24.8 & 23.0 & 21.3 \\
\hline & 15 & 40.0 & 38.2 & 36.5 & 34.7 & 33.0 & 31.3 & 29.5 & 27.8 & 26.1 & 24.3 & 22.6 & 20.8 & 19.1 \\
\hline & 10 & 37.3 & 35.6 & 33.9 & 32.1 & 30.4 & 28.6 & 26.9 & 25.2 & 23.4 & 21.7 & 20.0 & 18.2 & 16.5 \\
\hline & 5 & 33.4 & 31.7 & 29.9 & 28.2 & 26.4 & 24.7 & 23.0 & 21.2 & 19.5 & 17.7 & 16.0 & 14.3 & 12.5 \\
\hline \multirow{13}{*}{ 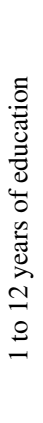 } & 95 & 51.5 & 49.7 & 48.0 & 46.3 & 44.5 & 42.8 & 41.0 & 39.3 & 37.6 & 35.8 & 34.1 & 32.4 & 30.6 \\
\hline & 90 & 47.5 & 45.8 & 44.1 & 42.3 & 40.6 & 38.8 & 37.1 & 35.4 & 33.6 & 31.9 & 30.2 & 28.4 & 26.7 \\
\hline & 85 & 44.9 & 43.2 & 41.4 & 39.7 & 38.0 & 36.2 & 34.5 & 32.7 & 31.0 & 29.3 & 27.5 & 25.8 & 24.0 \\
\hline & 80 & 42.7 & 41.0 & 39.2 & 37.5 & 35.8 & 34.0 & 32.3 & 30.5 & 28.8 & 27.1 & 25.3 & 23.6 & 21.9 \\
\hline & 70 & 39.2 & 37.5 & 35.7 & 34.0 & 32.3 & 30.5 & 28.8 & 27.0 & 25.3 & 23.6 & 21.8 & 20.1 & 18.3 \\
\hline & 60 & 36.2 & 34.5 & 32.8 & 31.0 & 29.3 & 27.6 & 25.8 & 24.1 & 22.3 & 20.6 & 18.9 & 17.1 & 15.4 \\
\hline & 50 & 33.5 & 31.8 & 30.0 & 28.3 & 26.6 & 24.8 & 23.1 & 21.3 & 19.6 & 17.9 & 16.1 & 14.4 & 12.7 \\
\hline & 40 & 30.8 & 29.0 & 27.3 & 25.6 & 23.8 & 22.1 & 20.3 & 18.6 & 16.9 & 15.1 & 13.4 & 11.7 & 9.9 \\
\hline & 30 & 27.8 & 26.1 & 24.3 & 22.6 & 20.9 & 19.1 & 17.4 & 15.6 & 13.9 & 12.2 & 10.4 & 8.7 & 7.0 \\
\hline & 20 & 24.3 & 22.6 & 20.8 & 19.1 & 17.4 & 15.6 & 13.9 & 12.1 & 10.4 & 8.7 & 6.9 & 5.2 & 3.4 \\
\hline & 15 & 22.1 & 20.4 & 18.6 & 16.9 & 15.2 & 13.4 & 11.7 & 9.9 & 8.2 & 6.5 & 4.7 & 3.0 & 1.3 \\
\hline & 10 & 19.5 & 17.7 & 16.0 & 14.3 & 12.5 & 10.8 & 9.1 & 7.3 & 5.6 & 3.8 & 2.1 & 0.4 & - \\
\hline & 5 & 15.5 & 13.8 & 12.1 & 10.3 & 8.6 & 6.9 & 5.1 & 3.4 & 1.6 & - & - & - & - \\
\hline
\end{tabular}

Table A6

Normative data for the SDMT stratified by education levels for GUATEMALA

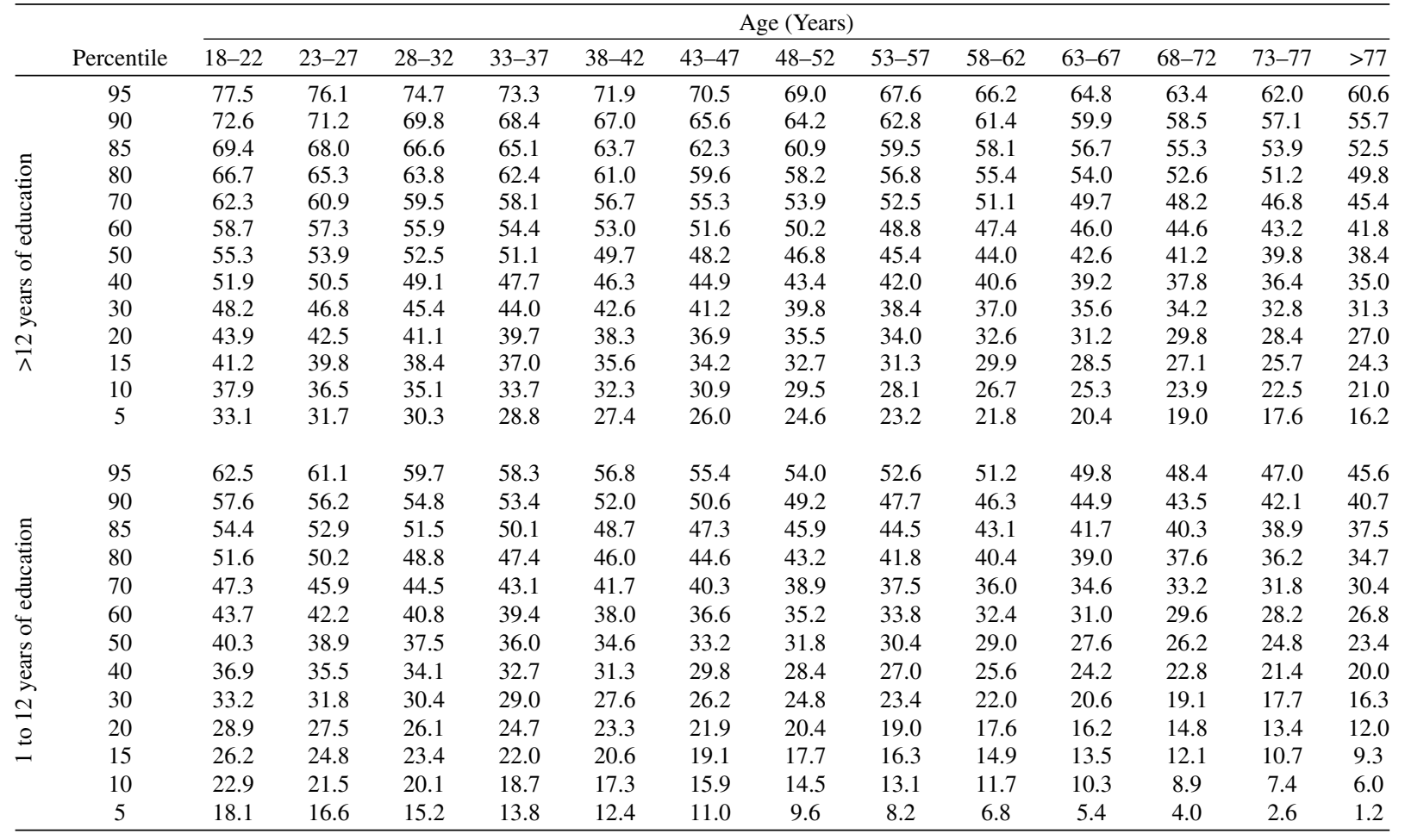


Table A7

Normative data for the SDMT stratified by age and education levels for HONDURAS

\begin{tabular}{|c|c|c|c|c|c|c|c|c|c|c|c|c|c|c|}
\hline & \multirow[b]{2}{*}{ Percentile } & \multicolumn{13}{|c|}{ Age (Years) } \\
\hline & & $18-22$ & $23-27$ & $28-32$ & $33-37$ & $38-42$ & $43-47$ & $48-52$ & $53-57$ & $58-62$ & $63-67$ & $68-72$ & $73-77$ & $>77$ \\
\hline \multirow{13}{*}{ 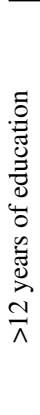 } & 95 & 61.9 & 60.2 & 58.6 & 56.9 & 55.3 & 53.6 & 52.0 & 50.3 & 48.6 & 47.0 & 45.3 & 43.7 & 42.0 \\
\hline & 90 & 58.3 & 56.7 & 55.0 & 53.4 & 51.7 & 50.1 & 48.4 & 46.7 & 45.1 & 43.4 & 41.8 & 40.1 & 38.5 \\
\hline & 85 & 56.0 & 54.3 & 52.7 & 51.0 & 49.3 & 47.7 & 46.0 & 44.4 & 42.7 & 41.1 & 39.4 & 37.7 & 36.1 \\
\hline & 80 & 54.0 & 52.3 & 50.7 & 49.0 & 47.4 & 45.7 & 44.1 & 42.4 & 40.7 & 39.1 & 37.4 & 35.8 & 34.1 \\
\hline & 70 & 50.8 & 49.2 & 47.5 & 45.9 & 44.2 & 42.6 & 40.9 & 39.2 & 37.6 & 35.9 & 34.3 & 32.6 & 30.9 \\
\hline & 60 & 48.2 & 46.5 & 44.9 & 43.2 & 41.5 & 39.9 & 38.2 & 36.6 & 34.9 & 33.3 & 31.6 & 29.9 & 28.3 \\
\hline & 50 & 45.7 & 44.0 & 42.4 & 40.7 & 39.1 & 37.4 & 35.8 & 34.1 & 32.4 & 30.8 & 29.1 & 27.5 & 25.8 \\
\hline & 40 & 43.2 & 41.6 & 39.9 & 38.3 & 36.6 & 34.9 & 33.3 & 31.6 & 30.0 & 28.3 & 26.7 & 25.0 & 23.3 \\
\hline & 30 & 40.6 & 38.9 & 37.3 & 35.6 & 33.9 & 32.3 & 30.6 & 29.0 & 27.3 & 25.7 & 24.0 & 22.3 & 20.7 \\
\hline & 20 & 37.4 & 35.8 & 34.1 & 32.4 & 30.8 & 29.1 & 27.5 & 25.8 & 24.1 & 22.5 & 20.8 & 19.2 & 17.5 \\
\hline & 15 & 35.4 & 33.8 & 32.1 & 30.5 & 28.8 & 27.1 & 25.5 & 23.8 & 22.2 & 20.5 & 18.9 & 17.2 & 15.5 \\
\hline & 10 & 33.1 & 31.4 & 29.7 & 28.1 & 26.4 & 24.8 & 23.1 & 21.5 & 19.8 & 18.1 & 16.5 & 14.8 & 13.2 \\
\hline & 5 & 29.5 & 27.9 & 26.2 & 24.5 & 22.9 & 21.2 & 19.6 & 17.9 & 16.2 & 14.6 & 12.9 & 11.3 & 9.6 \\
\hline \multirow{13}{*}{ 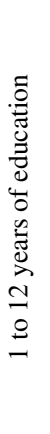 } & 95 & 47.4 & 45.8 & 44.1 & 42.4 & 40.8 & 39.1 & 37.5 & 35.8 & 34.2 & 32.5 & 30.8 & 29.2 & 27.5 \\
\hline & 90 & 43.9 & 42.2 & 40.5 & 38.9 & 37.2 & 35.6 & 33.9 & 32.3 & 30.6 & 28.9 & 27.3 & 25.6 & 24.0 \\
\hline & 85 & 41.5 & 39.8 & 38.2 & 36.5 & 34.9 & 33.2 & 31.5 & 29.9 & 28.2 & 26.6 & 24.9 & 23.3 & 21.6 \\
\hline & 80 & 39.5 & 37.9 & 36.2 & 34.5 & 32.9 & 31.2 & 29.6 & 27.9 & 26.3 & 24.6 & 22.9 & 21.3 & 19.6 \\
\hline & 70 & 36.4 & 34.7 & 33.0 & 31.4 & 29.7 & 28.1 & 26.4 & 24.8 & 23.1 & 21.4 & 19.8 & 18.1 & 16.5 \\
\hline & 60 & 33.7 & 32.0 & 30.4 & 28.7 & 27.1 & 25.4 & 23.7 & 22.1 & 20.4 & 18.8 & 17.1 & 15.5 & 13.8 \\
\hline & 50 & 31.2 & 29.6 & 27.9 & 26.3 & 24.6 & 22.9 & 21.3 & 19.6 & 18.0 & 16.3 & 14.6 & 13.0 & 11.3 \\
\hline & 40 & 28.8 & 27.1 & 25.4 & 23.8 & 22.1 & 20.5 & 18.8 & 17.2 & 15.5 & 13.8 & 12.2 & 10.5 & 8.9 \\
\hline & 30 & 26.1 & 24.4 & 22.8 & 21.1 & 19.5 & 17.8 & 16.1 & 14.5 & 12.8 & 11.2 & 9.5 & 7.9 & 6.2 \\
\hline & 20 & 22.9 & 21.3 & 19.6 & 18.0 & 16.3 & 14.6 & 13.0 & 11.3 & 9.7 & 8.0 & 6.4 & 4.7 & 3.0 \\
\hline & 15 & 21.0 & 19.3 & 17.6 & 16.0 & 14.3 & 12.7 & 11.0 & 9.4 & 7.7 & 6.0 & 4.4 & 2.7 & 1.1 \\
\hline & 10 & 18.6 & 16.9 & 15.3 & 13.6 & 12.0 & 10.3 & 8.6 & 7.0 & 5.3 & 3.7 & 2.0 & 0.4 & - \\
\hline & 5 & 15.0 & 13.4 & 11.7 & 10.1 & 8.4 & 6.7 & 5.1 & 3.4 & 1.8 & 0.1 & - & - & - \\
\hline
\end{tabular}

Table A8

Normative data for the SDMT stratified by age and education levels for MEXICO

\begin{tabular}{|c|c|c|c|c|c|c|c|c|c|c|c|c|c|c|}
\hline & \multirow[b]{2}{*}{ Percentile } & \multicolumn{13}{|c|}{ Age (Years) } \\
\hline & & $18-22$ & $23-27$ & $28-32$ & $33-37$ & $38-42$ & $43-47$ & $48-52$ & $53-57$ & $58-62$ & $63-67$ & $68-72$ & $73-77$ & $>77$ \\
\hline \multirow{13}{*}{ 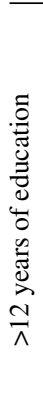 } & 95 & 77.3 & 75.0 & 72.6 & 70.2 & 67.9 & 65.5 & 63.2 & 60.8 & 58.5 & 56.1 & 53.8 & 51.4 & 49.0 \\
\hline & 90 & 73.0 & 70.6 & 68.3 & 65.9 & 63.6 & 61.2 & 58.9 & 56.5 & 54.1 & 51.8 & 49.4 & 47.1 & 44.7 \\
\hline & 85 & 70.1 & 67.7 & 65.4 & 63.0 & 60.7 & 58.3 & 56.0 & 53.6 & 51.3 & 48.9 & 46.5 & 44.2 & 41.8 \\
\hline & 80 & 67.7 & 65.3 & 63.0 & 60.6 & 58.3 & 55.9 & 53.6 & 51.2 & 48.9 & 46.5 & 44.1 & 41.8 & 39.4 \\
\hline & 70 & 63.9 & 61.5 & 59.1 & 56.8 & 54.4 & 52.1 & 49.7 & 47.4 & 45.0 & 42.7 & 40.3 & 37.9 & 35.6 \\
\hline & 60 & 60.6 & 58.3 & 55.9 & 53.5 & 51.2 & 48.8 & 46.5 & 44.1 & 41.8 & 39.4 & 37.1 & 34.7 & 32.3 \\
\hline & 50 & 57.6 & 55.3 & 52.9 & 50.5 & 48.2 & 45.8 & 43.5 & 41.1 & 38.8 & 36.4 & 34.1 & 31.7 & 29.3 \\
\hline & 40 & 54.6 & 52.3 & 49.9 & 47.5 & 45.2 & 42.8 & 40.5 & 38.1 & 35.8 & 33.4 & 31.0 & 28.7 & 26.3 \\
\hline & 30 & 51.4 & 49.0 & 46.7 & 44.3 & 41.9 & 39.6 & 37.2 & 34.9 & 32.5 & 30.2 & 27.8 & 25.5 & 23.1 \\
\hline & 20 & 47.5 & 45.2 & 42.8 & 40.5 & 38.1 & 35.7 & 33.4 & 31.0 & 28.7 & 26.3 & 24.0 & 21.6 & 19.3 \\
\hline & 15 & 45.1 & 42.8 & 40.4 & 38.0 & 35.7 & 33.3 & 31.0 & 28.6 & 26.3 & 23.9 & 21.6 & 19.2 & 16.8 \\
\hline & 10 & 42.2 & 39.9 & 37.5 & 35.2 & 32.8 & 30.5 & 28.1 & 25.7 & 23.4 & 21.0 & 18.7 & 16.3 & 14.0 \\
\hline & 5 & 37.9 & 35.6 & 33.2 & 30.8 & 28.5 & 26.1 & 23.8 & 21.4 & 19.1 & 16.7 & 14.4 & 12.0 & 9.6 \\
\hline \multirow{13}{*}{ 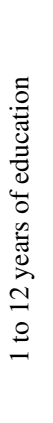 } & 95 & 71.1 & 68.7 & 66.3 & 64.0 & 61.7 & 59.3 & 56.9 & 54.6 & 52.2 & 49.9 & 47.5 & 45.1 & 42.8 \\
\hline & 90 & 66.8 & 64.4 & 62.0 & 59.7 & 57.3 & 55.0 & 52.6 & 50.3 & 47.9 & 45.6 & 43.2 & 40.8 & 38.5 \\
\hline & 85 & 63.9 & 61.5 & 59.2 & 56.8 & 54.4 & 52.1 & 49.7 & 47.4 & 45.0 & 42.7 & 40.3 & 38.0 & 35.6 \\
\hline & 80 & 61.5 & 59.1 & 56.8 & 54.4 & 52.0 & 49.7 & 47.3 & 45.0 & 42.6 & 40.3 & 37.9 & 35.6 & 33.2 \\
\hline & 70 & 57.6 & 55.3 & 52.9 & 50.6 & 48.2 & 45.8 & 43.5 & 41.1 & 38.8 & 36.4 & 34.1 & 31.7 & 29.4 \\
\hline & 60 & 54.4 & 52.0 & 49.7 & 47.3 & 45.0 & 42.6 & 40.2 & 37.9 & 35.5 & 33.2 & 30.8 & 28.5 & 26.1 \\
\hline & 50 & 51.4 & 49.0 & 46.7 & 44.3 & 42.0 & 39.6 & 37.2 & 34.9 & 32.5 & 30.2 & 27.8 & 25.5 & 23.1 \\
\hline & 40 & 48.4 & 46.0 & 43.7 & 41.3 & 39.0 & 36.6 & 34.2 & 31.9 & 29.5 & 27.2 & 24.8 & 22.5 & 20.1 \\
\hline & 30 & 45.1 & 42.8 & 40.4 & 38.1 & 35.7 & 33.4 & 31.0 & 28.6 & 26.3 & 23.9 & 21.6 & 19.2 & 16.9 \\
\hline & 20 & 41.3 & 38.9 & 36.6 & 34.2 & 31.9 & 29.5 & 27.2 & 24.8 & 22.4 & 20.1 & 17.7 & 15.4 & 13.0 \\
\hline & 15 & 38.9 & 36.5 & 34.2 & 31.8 & 29.5 & 27.1 & 24.8 & 22.4 & 20.0 & 17.7 & 15.3 & 13.0 & 10.6 \\
\hline & 10 & 36.0 & 33.6 & 31.3 & 28.9 & 26.6 & 24.2 & 21.9 & 19.5 & 17.2 & 14.8 & 12.4 & 10.1 & 7.7 \\
\hline & 5 & 31.7 & 29.3 & 27.0 & 24.6 & 22.3 & 19.9 & 17.5 & 15.2 & 12.8 & 10.5 & 8.1 & 5.8 & 3.4 \\
\hline
\end{tabular}


Table A9

Normative data for the SDMT stratified by age and education levels for PARAGUAY

\begin{tabular}{|c|c|c|c|c|c|c|c|c|c|c|c|c|c|c|}
\hline & \multirow[b]{2}{*}{ Percentile } & \multicolumn{13}{|c|}{ Age (Years) } \\
\hline & & $18-22$ & $23-27$ & $28-32$ & $33-37$ & $38-42$ & $43-47$ & $48-52$ & $53-57$ & $58-62$ & $63-67$ & $68-72$ & $73-77$ & $>77$ \\
\hline \multirow{13}{*}{ 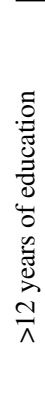 } & 95 & 49.5 & 48.4 & 47.3 & 46.1 & 45.0 & 43.9 & 42.7 & 41.6 & 40.5 & 39.4 & 38.2 & 37.1 & 36.0 \\
\hline & 90 & 47.0 & 45.9 & 44.8 & 43.7 & 42.5 & 41.4 & 40.3 & 39.1 & 38.0 & 36.9 & 35.8 & 34.6 & 33.5 \\
\hline & 85 & 45.4 & 44.3 & 43.1 & 42.0 & 40.9 & 39.8 & 38.6 & 37.5 & 36.4 & 35.2 & 34.1 & 33.0 & 31.9 \\
\hline & 80 & 44.0 & 42.9 & 41.8 & 40.6 & 39.5 & 38.4 & 37.2 & 36.1 & 35.0 & 33.9 & 32.7 & 31.6 & 30.5 \\
\hline & 70 & 41.8 & 40.7 & 39.6 & 38.4 & 37.3 & 36.2 & 35.1 & 33.9 & 32.8 & 31.7 & 30.5 & 29.4 & 28.3 \\
\hline & 60 & 40.0 & 38.8 & 37.7 & 36.6 & 35.5 & 34.3 & 33.2 & 32.1 & 30.9 & 29.8 & 28.7 & 27.6 & 26.4 \\
\hline & 50 & 38.3 & 37.1 & 36.0 & 34.9 & 33.7 & 32.6 & 31.5 & 30.4 & 29.2 & 28.1 & 27.0 & 25.8 & 24.7 \\
\hline & 40 & 36.5 & 35.4 & 34.3 & 33.2 & 32.0 & 30.9 & 29.8 & 28.6 & 27.5 & 26.4 & 25.3 & 24.1 & 23.0 \\
\hline & 30 & 34.7 & 33.6 & 32.4 & 31.3 & 30.2 & 29.0 & 27.9 & 26.8 & 25.7 & 24.5 & 23.4 & 22.3 & 21.1 \\
\hline & 20 & 32.5 & 31.4 & 30.2 & 29.1 & 28.0 & 26.8 & 25.7 & 24.6 & 23.5 & 22.3 & 21.2 & 20.1 & 18.9 \\
\hline & 15 & 31.1 & 30.0 & 28.9 & 27.7 & 26.6 & 25.5 & 24.3 & 23.2 & 22.1 & 21.0 & 19.8 & 18.7 & 17.6 \\
\hline & 10 & 29.5 & 28.3 & 27.2 & 26.1 & 25.0 & 23.8 & 22.7 & 21.6 & 20.4 & 19.3 & 18.2 & 17.1 & 15.9 \\
\hline & 5 & 27.0 & 25.9 & 24.7 & 23.6 & 22.5 & 21.4 & 20.2 & 19.1 & 18.0 & 16.8 & 15.7 & 14.6 & 13.5 \\
\hline \multirow{13}{*}{ 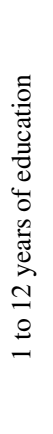 } & 95 & 39.3 & 38.1 & 37.0 & 35.9 & 34.8 & 33.6 & 32.5 & 31.4 & 30.2 & 29.1 & 28.0 & 26.9 & 25.7 \\
\hline & 90 & 36.8 & 35.7 & 34.5 & 33.4 & 32.3 & 31.2 & 30.0 & 28.9 & 27.8 & 26.6 & 25.5 & 24.4 & 23.3 \\
\hline & 85 & 35.1 & 34.0 & 32.9 & 31.8 & 30.6 & 29.5 & 28.4 & 27.3 & 26.1 & 25.0 & 23.9 & 22.7 & 21.6 \\
\hline & 80 & 33.8 & 32.6 & 31.5 & 30.4 & 29.3 & 28.1 & 27.0 & 25.9 & 24.8 & 23.6 & 22.5 & 21.4 & 20.2 \\
\hline & 70 & 31.6 & 30.5 & 29.3 & 28.2 & 27.1 & 25.9 & 24.8 & 23.7 & 22.6 & 21.4 & 20.3 & 19.2 & 18.0 \\
\hline & 60 & 29.7 & 28.6 & 27.5 & 26.3 & 25.2 & 24.1 & 23.0 & 21.8 & 20.7 & 19.6 & 18.4 & 17.3 & 16.2 \\
\hline & 50 & 28.0 & 26.9 & 25.8 & 24.6 & 23.5 & 22.4 & 21.2 & 20.1 & 19.0 & 17.9 & 16.7 & 15.6 & 14.5 \\
\hline & 40 & 26.3 & 25.2 & 24.0 & 22.9 & 21.8 & 20.7 & 19.5 & 18.4 & 17.3 & 16.1 & 15.0 & 13.9 & 12.8 \\
\hline & 30 & 24.4 & 23.3 & 22.2 & 21.1 & 19.9 & 18.8 & 17.7 & 16.5 & 15.4 & 14.3 & 13.2 & 12.0 & 10.9 \\
\hline & 20 & 22.2 & 21.1 & 20.0 & 18.9 & 17.7 & 16.6 & 15.5 & 14.3 & 13.2 & 12.1 & 11.0 & 9.8 & 8.7 \\
\hline & 15 & 20.9 & 19.7 & 18.6 & 17.5 & 16.4 & 15.2 & 14.1 & 13.0 & 11.8 & 10.7 & 9.6 & 8.5 & 7.3 \\
\hline & 10 & 19.2 & 18.1 & 17.0 & 15.8 & 14.7 & 13.6 & 12.5 & 11.3 & 10.2 & 9.1 & 7.9 & 6.8 & 5.7 \\
\hline & 5 & 16.7 & 15.6 & 14.5 & 13.4 & 12.2 & 11.1 & 10.0 & 8.9 & 7.7 & 6.6 & 5.5 & 4.3 & 3.2 \\
\hline
\end{tabular}

Table A10

Normative data for the SDMT stratified by age and education levels for PERU

\begin{tabular}{|c|c|c|c|c|c|c|c|c|c|c|c|c|c|c|}
\hline & \multirow[b]{2}{*}{ Percentile } & \multicolumn{13}{|c|}{ Age (Years) } \\
\hline & & $18-22$ & $23-27$ & $28-32$ & $33-37$ & $38-42$ & $43-47$ & $48-52$ & $53-57$ & $58-62$ & $63-67$ & $68-72$ & $73-77$ & $>77$ \\
\hline \multirow{13}{*}{ 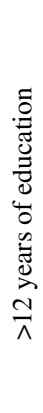 } & 95 & 69.6 & 67.6 & 65.5 & 63.5 & 61.5 & 59.5 & 57.5 & 55.5 & 53.4 & 51.4 & 49.4 & 47.4 & 45.4 \\
\hline & 90 & 66.2 & 64.1 & 62.1 & 60.1 & 58.1 & 56.1 & 54.1 & 52.0 & 50.0 & 48.0 & 46.0 & 44.0 & 42.0 \\
\hline & 85 & 63.9 & 61.9 & 59.9 & 57.8 & 55.8 & 53.8 & 51.8 & 49.8 & 47.7 & 45.7 & 43.7 & 41.7 & 39.7 \\
\hline & 80 & 62.0 & 60.0 & 58.0 & 55.9 & 53.9 & 51.9 & 49.9 & 47.9 & 45.9 & 43.8 & 41.8 & 39.8 & 37.8 \\
\hline & 70 & 59.0 & 56.9 & 54.9 & 52.9 & 50.9 & 48.9 & 46.9 & 44.8 & 42.8 & 40.8 & 38.8 & 36.8 & 34.7 \\
\hline & 60 & 56.4 & 54.4 & 52.4 & 50.3 & 48.3 & 46.3 & 44.3 & 42.3 & 40.3 & 38.2 & 36.2 & 34.2 & 32.2 \\
\hline & 50 & 54.0 & 52.0 & 50.0 & 48.0 & 46.0 & 43.9 & 41.9 & 39.9 & 37.9 & 35.9 & 33.8 & 31.8 & 29.8 \\
\hline & 40 & 51.7 & 49.6 & 47.6 & 45.6 & 43.6 & 41.6 & 39.5 & 37.5 & 35.5 & 33.5 & 31.5 & 29.5 & 27.4 \\
\hline & 30 & 49.1 & 47.1 & 45.1 & 43.0 & 41.0 & 39.0 & 37.0 & 35.0 & 33.0 & 30.9 & 28.9 & 26.9 & 24.9 \\
\hline & 20 & 46.1 & 44.0 & 42.0 & 40.0 & 38.0 & 36.0 & 34.0 & 31.9 & 29.9 & 27.9 & 25.9 & 23.9 & 21.8 \\
\hline & 15 & 44.2 & 42.1 & 40.1 & 38.1 & 36.1 & 34.1 & 32.1 & 30.0 & 28.0 & 26.0 & 24.0 & 22.0 & 19.9 \\
\hline & 10 & 41.9 & 39.9 & 37.8 & 35.8 & 33.8 & 31.8 & 29.8 & 27.8 & 25.7 & 23.7 & 21.7 & 19.7 & 17.7 \\
\hline & 5 & 38.5 & 36.4 & 34.4 & 32.4 & 30.4 & 28.4 & 26.4 & 24.3 & 22.3 & 20.3 & 18.3 & 16.3 & 14.3 \\
\hline \multirow{13}{*}{ 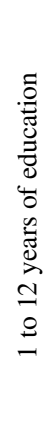 } & 95 & 60.2 & 58.2 & 56.1 & 54.1 & 52.1 & 50.1 & 48.1 & 46.1 & 44.0 & 42.0 & 40.0 & 38.0 & 36.0 \\
\hline & 90 & 56.8 & 54.7 & 52.7 & 50.7 & 48.7 & 46.7 & 44.7 & 42.6 & 40.6 & 38.6 & 36.6 & 34.6 & 32.6 \\
\hline & 85 & 54.5 & 52.5 & 50.5 & 48.4 & 46.4 & 44.4 & 42.4 & 40.4 & 38.4 & 36.3 & 34.3 & 32.3 & 30.3 \\
\hline & 80 & 52.6 & 50.6 & 48.6 & 46.5 & 44.5 & 42.5 & 40.5 & 38.5 & 36.5 & 34.4 & 32.4 & 30.4 & 28.4 \\
\hline & 70 & 49.6 & 47.5 & 45.5 & 43.5 & 41.5 & 39.5 & 37.5 & 35.4 & 33.4 & 31.4 & 29.4 & 27.4 & 25.3 \\
\hline & 60 & 47.0 & 45.0 & 43.0 & 40.9 & 38.9 & 36.9 & 34.9 & 32.9 & 30.9 & 28.8 & 26.8 & 24.8 & 22.8 \\
\hline & 50 & 44.6 & 42.6 & 40.6 & 38.6 & 36.6 & 34.5 & 32.5 & 30.5 & 28.5 & 26.5 & 24.4 & 22.4 & 20.4 \\
\hline & 40 & 42.3 & 40.2 & 38.2 & 36.2 & 34.2 & 32.2 & 30.1 & 28.1 & 26.1 & 24.1 & 22.1 & 20.1 & 18.0 \\
\hline & 30 & 39.7 & 37.7 & 35.7 & 33.6 & 31.6 & 29.6 & 27.6 & 25.6 & 23.6 & 21.5 & 19.5 & 17.5 & 15.5 \\
\hline & 20 & 36.7 & 34.6 & 32.6 & 30.6 & 28.6 & 26.6 & 24.6 & 22.5 & 20.5 & 18.5 & 16.5 & 14.5 & 12.4 \\
\hline & 15 & 34.8 & 32.7 & 30.7 & 28.7 & 26.7 & 24.7 & 22.7 & 20.6 & 18.6 & 16.6 & 14.6 & 12.6 & 10.5 \\
\hline & 10 & 32.5 & 30.5 & 28.4 & 26.4 & 24.4 & 22.4 & 20.4 & 18.4 & 16.3 & 14.3 & 12.3 & 10.3 & 8.3 \\
\hline & 5 & 29.1 & 27.0 & 25.0 & 23.0 & 21.0 & 19.0 & 17.0 & 14.9 & 12.9 & 10.9 & 8.9 & 6.9 & 4.9 \\
\hline
\end{tabular}


Table A11

Normative data for the SDMT stratified by age and education levels for PUERTO RICO

\begin{tabular}{|c|c|c|c|c|c|c|c|c|c|c|c|c|c|c|}
\hline & \multirow[b]{2}{*}{ Percentile } & \multicolumn{13}{|c|}{ Age (Years) } \\
\hline & & $18-22$ & $23-27$ & $28-32$ & $33-37$ & $38-42$ & $43-47$ & $48-52$ & $53-57$ & $58-62$ & $63-67$ & $68-72$ & $73-77$ & $>77$ \\
\hline \multirow{13}{*}{ 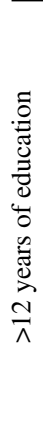 } & 95 & 77.8 & 75.1 & 72.5 & 69.8 & 67.1 & 64.5 & 61.8 & 59.1 & 56.5 & 53.8 & 51.1 & 48.5 & 45.8 \\
\hline & 90 & 73.7 & 71.1 & 68.4 & 65.7 & 63.0 & 60.4 & 57.7 & 55.0 & 52.4 & 49.7 & 47.0 & 44.4 & 41.7 \\
\hline & 85 & 71.0 & 68.3 & 65.7 & 63.0 & 60.3 & 57.6 & 55.0 & 52.3 & 49.6 & 47.0 & 44.3 & 41.6 & 39.0 \\
\hline & 80 & 68.7 & 66.0 & 63.4 & 60.7 & 58.0 & 55.4 & 52.7 & 50.0 & 47.4 & 44.7 & 42.0 & 39.4 & 36.7 \\
\hline & 70 & 65.1 & 62.4 & 59.7 & 57.1 & 54.4 & 51.7 & 49.1 & 46.4 & 43.7 & 41.1 & 38.4 & 35.7 & 33.1 \\
\hline & 60 & 62.0 & 59.3 & 56.7 & 54.0 & 51.3 & 48.7 & 46.0 & 43.3 & 40.7 & 38.0 & 35.3 & 32.6 & 30.0 \\
\hline & 50 & 59.2 & 56.5 & 53.8 & 51.1 & 48.5 & 45.8 & 43.1 & 40.5 & 37.8 & 35.1 & 32.5 & 29.8 & 27.1 \\
\hline & 40 & 56.3 & 53.6 & 51.0 & 48.3 & 45.6 & 43.0 & 40.3 & 37.6 & 35.0 & 32.3 & 29.6 & 27.0 & 24.3 \\
\hline & 30 & 53.2 & 50.6 & 47.9 & 45.2 & 42.6 & 39.9 & 37.2 & 34.6 & 31.9 & 29.2 & 26.6 & 23.9 & 21.2 \\
\hline & 20 & 49.6 & 46.9 & 44.3 & 41.6 & 38.9 & 36.3 & 33.6 & 30.9 & 28.2 & 25.6 & 22.9 & 20.2 & 17.6 \\
\hline & 15 & 47.3 & 44.6 & 42.0 & 39.3 & 36.6 & 34.0 & 31.3 & 28.6 & 26.0 & 23.3 & 20.6 & 18.0 & 15.3 \\
\hline & 10 & 44.6 & 41.9 & 39.2 & 36.6 & 33.9 & 31.2 & 28.6 & 25.9 & 23.2 & 20.6 & 17.9 & 15.2 & 12.6 \\
\hline & 5 & 40.5 & 37.8 & 35.2 & 32.5 & 29.8 & 27.1 & 24.5 & 21.8 & 19.1 & 16.5 & 13.8 & 11.1 & 8.5 \\
\hline \multirow{13}{*}{ 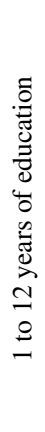 } & 95 & 72.8 & 70.1 & 67.5 & 64.8 & 62.1 & 59.5 & 56.8 & 54.1 & 51.5 & 48.8 & 46.1 & 43.5 & 40.8 \\
\hline & 90 & 68.7 & 66.0 & 63.4 & 60.7 & 58.0 & 55.4 & 52.7 & 50.0 & 47.4 & 44.7 & 42.0 & 39.4 & 36.7 \\
\hline & 85 & 66.0 & 63.3 & 60.6 & 58.0 & 55.3 & 52.6 & 50.0 & 47.3 & 44.6 & 42.0 & 39.3 & 36.6 & 34.0 \\
\hline & 80 & 63.7 & 61.0 & 58.4 & 55.7 & 53.0 & 50.4 & 47.7 & 45.0 & 42.4 & 39.7 & 37.0 & 34.4 & 31.7 \\
\hline & 70 & 60.1 & 57.4 & 54.7 & 52.1 & 49.4 & 46.7 & 44.1 & 41.4 & 38.7 & 36.0 & 33.4 & 30.7 & 28.0 \\
\hline & 60 & 57.0 & 54.3 & 51.7 & 49.0 & 46.3 & 43.6 & 41.0 & 38.3 & 35.6 & 33.0 & 30.3 & 27.6 & 25.0 \\
\hline & 50 & 54.1 & 51.5 & 48.8 & 46.1 & 43.5 & 40.8 & 38.1 & 35.5 & 32.8 & 30.1 & 27.5 & 24.8 & 22.1 \\
\hline & 40 & 51.3 & 48.6 & 46.0 & 43.3 & 40.6 & 38.0 & 35.3 & 32.6 & 30.0 & 27.3 & 24.6 & 21.9 & 19.3 \\
\hline & 30 & 48.2 & 45.6 & 42.9 & 40.2 & 37.6 & 34.9 & 32.2 & 29.5 & 26.9 & 24.2 & 21.5 & 18.9 & 16.2 \\
\hline & 20 & 44.6 & 41.9 & 39.2 & 36.6 & 33.9 & 31.2 & 28.6 & 25.9 & 23.2 & 20.6 & 17.9 & 15.2 & 12.6 \\
\hline & 15 & 42.3 & 39.6 & 37.0 & 34.3 & 31.6 & 29.0 & 26.3 & 23.6 & 21.0 & 18.3 & 15.6 & 13.0 & 10.3 \\
\hline & 10 & 39.6 & 36.9 & 34.2 & 31.6 & 28.9 & 26.2 & 23.6 & 20.9 & 18.2 & 15.6 & 12.9 & 10.2 & 7.6 \\
\hline & 5 & 35.5 & 32.8 & 30.1 & 27.5 & 24.8 & 22.1 & 19.5 & 16.8 & 14.1 & 11.5 & 8.8 & 6.1 & 3.5 \\
\hline
\end{tabular}

